\title{
Essential inpatient otolaryngology: what COVID-19 has revealed
}

\author{
Andre Shomorony ${ }^{1}$ (1) $\cdot$ Alexander Chern ${ }^{1} \cdot$ Sallie M. Long ${ }^{1} \cdot$ Noah Z. Feit $^{1} \cdot$ Sarita S. Ballakur $^{1} \cdot$ Mariam Gadjiko $^{1}$. \\ Katie Liu ${ }^{1}$ - Daniel A. Skaf ${ }^{1}$. Andrew B. Tassler ${ }^{1}$. Anthony P. Sclafani ${ }^{1}$
}

Received: 24 April 2021 / Accepted: 25 June 2021 / Published online: 10 July 2021

(C) The Author(s), under exclusive licence to Springer-Verlag GmbH Germany, part of Springer Nature 2021

\begin{abstract}
Purpose To identify areas of critical otolaryngology contributions to inpatient care resistant to disruption by the COVID-19 pandemic.

Methods Medical records of 614 otolaryngology consults seen between January and June of 2019 and 602 seen between January and June of 2020 were reviewed. Extracted data included patient demographics, SARS-CoV-2 status, medical comorbidities, consult location, consult category, reason for consult, procedures performed, and overall outcome. Prevalence of data items was compared using $t$ tests and Chi-squared tests.

Results The number of monthly consults to the otolaryngology service remained approximately stable after the onset of the COVID-19 pandemic. However, there was a substantial increase in ICU consults and a decrease in ER and floor consults. The proportion of otology, rhinology, and head and neck consults decreased while that of airway consults-most of which were tracheostomy-related-greatly increased. While the top ten reasons for consult remained essentially the same, they dramatically increased as a percentage of consults during COVID-19 (55-92\%), whereas there was a dramatic decrease in the proportion of less frequent consults.

Conclusion The changes in otolaryngology consultation patterns seen after the onset of the pandemic are multifactorial, but may be attributed to novel pathologies, attitudes, and policies. Nonetheless, these patterns reveal that a set of core otolaryngologic issues, including acute airway issues, head and neck lesions, severe sinusitis and epistaxis, are essential and need to be addressed in the inpatient setting, whereas the significant drop in other consults suggests that they may be appropriately managed on an outpatient basis.
\end{abstract}

Keywords COVID-19 $\cdot$ Inpatient consults · Tracheotomy $\cdot$ Comprehensive otolaryngology $\cdot$ Clinical research

Andre Shomorony

ans9365@nyp.org

Alexander Chern alc9230@nyp.org

Sallie M. Long

sm19023@nyp.org

Noah Z. Feit

nzf2001@med.cornell.edu

Sarita S. Ballakur

ssb2005@med.cornell.edu

Mariam Gadjiko

mag2093@med.cornell.edu
Katie Liu

ka14009@med.cornell.edu

Daniel A. Skaf

das2081@med.cornell.edu

Andrew B. Tassler

ant9025@med.cornell.edu

Anthony P. Sclafani

ans9243@med.cornell.edu

1 Department of Otolaryngology-Head and Neck Surgery, NewYork-Presbyterian/Weill Cornell Medical Center, 1305 York Avenue, 5thFloor, New York, NY 10021, United States of America 


\section{Introduction}

Since first identified in December 2019 in Wuhan, China [1], coronavirus disease 2019 (COVID-19) has spread rapidly throughout the world. As of May 30, 2021, worldwide COVID-19 cases exceeded 170 million and deaths exceeded 3.5 million (deaths in the United States exceeded $5,90,000$. In the United States, New York City (NYC) bore the brunt of the initial wave of the pandemic as an early epicenter of COVID-19, which led to widespread consequences for NYC healthcare systems. The New York-Presbyterian (NYP) healthcare system is a large integrated academic healthcare system providing care to patients in ten acute care facilities throughout New York City and the surrounding region. At the peak of the "first wave" of COVID in New York (April 14, 2020), the NYP system (with a normal baseline of 526 intensive care and 3682 total acute care beds) had 2512 inpatients who tested positive for the virus responsible for COVID-19 (SARS-CoV-2), with 746 patients on mechanical ventilation [2,3]. To accommodate these numbers, a colossal effort was required, including massive reconfiguration of existing physical space and hospital services, reassignment of healthcare personnel, and the creation of large-scale field hospitals.

Of the healthcare personnel involved in the front lines of this crisis, otolaryngologists have been uniquely impacted by the pandemic and are integral to the management of patients with COVID-19. COVID-19 often presents with otolaryngologic symptoms, such as cough, rhinorrhea, sore throat, dyspnea, anosmia, and dysgeusia. Otolaryngologists are also frequently consulted for expertise in airway management; many patients with COVID-19 have required prolonged mechanical ventilation, resulting in primary services consulting otolaryngologists to perform and manage tracheotomies [4]. As such, COVID-19 has had a large impact on the practice of otolaryngology.

Prior to the pandemic, otolaryngologists were frequently called for inpatient consultations for a variety of pathologies, from chronic benign conditions (e.g., cerumen impaction) to acute, life-threatening airway emergencies. However, the COVID-19 pandemic changed the approach and practices towards otolaryngology inpatient consultations. Due to the high viral load in the nasal cavity and nasopharynx, physicians who participate in aerosolgenerating examinations and procedures are at increased risk for inadvertent viral inoculation; indeed, healthcare personnel such as otolaryngologists and anesthesiologists had a high rate of infection and death during the initial outbreak in Wuhan, China [1,5-7]. These aforementioned risks and concerns over lack of personal protective equipment (PPE) in the United States at the time led the American Academy of Otolaryngology-Head and Neck Surgery to release a position statement early in the pandemic (March 23, 2020) recommending that otolaryngologists limit patient care to time-sensitive and emergent problems and routinely use appropriate PPE when treating patients [8]. Other groups have proposed similar guidelines regarding triage and PPE for management of otolaryngology patients, and have reported changes in outpatient and operative otolaryngologic practice due to these guidelines [9-12]. However, to the best of our knowledge, no existing literature has characterized the impact of the pandemic on inpatient otolaryngologic consultations in the United States. Our objectives were to analyze the patterns of otolaryngology inpatient consultations before and during the COVID-19 pandemic, and to characterize consultations based on their urgency and relative occurrence. This study focuses on consultations to the otolaryngology service for patients admitted to a hospital unit or emergency roomi.e., consultations that did not happen in an outpatient or ambulatory setting. Using the conditions of the COVID-19 pandemic as a 'natural experiment,' we may better identify and focus on the inpatient complaints and pathologies requiring inpatient otolaryngology evaluations and those more appropriately managed on an outpatient basis.

\section{Methods}

\section{Data collection}

The electronic medical records of patients requiring inpatient otolaryngology consultations from January 1 to June 30, 2019 and from January 1 to June 30, 2020 were analyzed. All patient consults were performed at NewYorkPresbyterian/Weill Cornell Medical Center, an academic medical center and the largest individual facility in the NYP system. For each consult, extracted data included patient demographics, SARS-CoV-2 status, intubation status, medical comorbidities, consult location, consult category, reason for consult, procedures performed, disposition, and overall medical or surgical outcome. SARS-CoV-2 status was determined by a positive or negative test using the gold-standard reverse-transcriptase PCR test from nasopharyngeal swabs. The COVID-19 period examined in this study was defined as March 1-June 30, 2020, since the first documented case of COVID-19 in NYC was recorded on March 1, 2020 at an NYP facility and, by the end of June 2020, some restrictions on public gatherings in New York State had been partially relaxed. The remainder of the study period (January 1-June 30, 2019, and January 1-February 29, 2020) was considered the pre-COVID-19 period. The periods of January 1-June 30 of 2 consecutive years were also chosen to allow for an overall comparison of monthly consults in 2019 and 2020. 


\section{Labeling of consult data}

To ensure consistent classification and analysis, each consult was assigned to 1 of 6 different categories (otology, rhinology, oral cavity/oropharynx, airway, head and neck, facial trauma) and 1 of 44 different reasons for consult (Supplemental Materials-Item 1).

\section{Statistical analysis}

Data were analyzed using MATLAB 2020 (MathWorks, Natick, MA). The prevalence of each data item from otolaryngology consults, recorded for each month between
$1 / 1 / 2019$ and 6/30/2019 and between $1 / 1 / 20$ and 6/30/20 (data presented in Tables 1, 2 and Figs. 2-4A), was compared between the periods before and during the COVID19 pandemic and reported using means, standard deviations, and two-tailed $p$ values computed from Student's $t$ tests. This statistical test was selected given the large sample sizes and normal distribution of independently sampled data. Whenever single proportions of consults were compared (data presented in Figs. 4B, 5 and S1), Chi-squared $\left(\chi^{2}\right)$ tests were used. Throughout the paper, amounts of consults were expressed as either absolute numbers, when focusing on volume of consults, or percentages, when focusing on changes in the proportion of certain consult classifications.
Table 1 General demographics and health status

\begin{tabular}{lllc}
\hline & $\begin{array}{l}\text { PRE-COVID-19 (Jan-Jun } \\
\text { 2019, Jan-Feb 2020) }\end{array}$ & $\begin{array}{l}\text { During COVID-19 } \\
\text { (Mar-Jun 2020) }\end{array}$ & $p$ value \\
\hline Total number of consults & 833 & 383 & - \\
Consults per month (mean \pm SD) & $104.1 \pm 8.6$ & $95.8 \pm 16.9$ & 0.324 \\
Age, adults (mean \pm SD) & $59.1 \pm 3.1$ & $60.9 \pm 2.7$ & 0.389 \\
Age, pediatric patients (mean \pm SD) & $4.1 \pm 1.3$ & $2.7 \pm 0.7$ & 0.548 \\
Pediatric consults $(\%$, mean \pm SD) & $24.9 \pm 5.7$ & $11.9 \pm 9.8$ & 0.024 \\
Ratio M:F & $53: 47$ & $59: 41$ & - \\
SARS-CoV-2 positivity $(\%$, mean \pm SD) & 0 & $39.0 \pm 30.4$ & - \\
Intubation ${ }^{\text {a }} \%$, mean \pm SD) & $8.0 \pm 3.0$ & $35.5 \pm 30.3$ & 0.022 \\
Anticoagulation $(\%$, mean \pm SD) & $8.8 \pm 2.3$ & $29.8 \pm 13.3$ & 0.003 \\
Renal replacement therapy $(\%$, mean \pm SD) & $2.6 \pm 1.2$ & $11.4 \pm 6.5$ & 0.003 \\
\hline
\end{tabular}

Mean and standard deviation (SD) are calculated over the months indicated for each period, and \% refers to percentage of consults per month

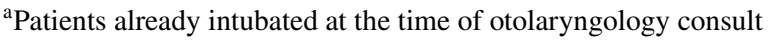

Table 2 Procedures performed before and during the COVID-19 pandemic

\begin{tabular}{llll}
\hline & $\begin{array}{l}\text { PRE-COVID-19 (Jan-Jun 2019, Jan-Feb } \\
\text { 2020) }\end{array}$ & During COVID-19 (Mar-Jun 2020) & $p$ value \\
\hline $\begin{array}{l}\text { Operative outcome by otolaryngology (\% of } \\
\text { consults, mean } \pm \text { SD) }\end{array}$ & $17.3 \pm 1.9$ & $31.2 \pm 21.0$ \\
$\begin{array}{l}\text { Bedside endoscopy (\% of consults, mean } \pm \\
\text { SD) }\end{array}$ & $55.1 \pm 6.1$ & $29.7 \pm 19.7$ \\
$\begin{array}{l}\text { Other bedside procedure (\% of consults, } \\
\text { mean } \pm \text { SD) }\end{array}$ & $13.7 \pm 3.8$ & $14.4 \pm 4.5$ & 0.122 \\
$\begin{array}{l}\text { Top five most common bedside procedures } \\
\text { (average number per month) }\end{array}$ & $\begin{array}{l}\text { Control of epistaxis (6.9) } \\
\text { Control of OC/OP bleeding (2.3) }\end{array}$ & $\begin{array}{l}\text { Control of epistaxis (5.5) } \\
\text { NG tube placement (1.1) } \\
\text { Repair of laceration (1.1) } \\
\text { Replacement of tracheostomy tube (0.9) }\end{array}$ & $\begin{array}{l}\text { Control of OC/OP bleeding }(2.0) \\
\text { Repair of laceration }(0.8)\end{array}$ \\
\hline
\end{tabular}

Operative outcomes, bedside endoscopies and other bedside procedures do not overlap. The top five most common bedside procedures are expressed as average number of procedures per month, and are accounted for in 'Other bedside procedure.' Mean and standard deviation (SD) are calculated over the months indicated for each period, and \% refers to percentage of consults per month. OC/OP, oral cavity/oropharynx; NG tube, nasogastric tube 


\section{Results}

\section{General trends}

There were 614 otolaryngology consults from 2019 and 602 consults from 2020 available for review. A comparison of consults during the first 6 months of 2019 and the first 6 months of 2020 revealed a similar overall number of monthly consults. However, the absolute number of monthly consults acutely dropped in March 2020, at the onset of the pandemic, and similarly increased in May 2020, with a rising need for tracheostomies in SARS-CoV-2-positive patients (Fig. 1). The number of monthly SARS-CoV-2-negative consults in 2020 reached its lowest point in April (19 consults or $18.6 \%$ of total consults) and approached its prepandemic baseline by June. Approximately $77 \%$ of SARSCoV-2-positive consults were tracheostomy-related consults (TRC), including both requests for tracheostomy and evaluation of tracheostomy-related issues. The number of SARSCoV-2-negative TRCs remained approximately stable from 2019 to 2020.

\section{Demographics}

The average number of otolaryngology consults per month before the pandemic (January-June 2019, and January-February 2020) and during the pandemic (March-June 2020) was essentially unchanged (104.1 vs. $95.8, p=0.324$; Table 1). Approximately $43 \%$ of consult patients during the pandemic were SARS-CoV-2-positive (having had at least one positive SARS-CoV-2 PCR test during their hospitalization), corresponding to a monthly mean of 39.0 consults. There was a significant increase in consult patients who were intubated during the pandemic compared to the prepandemic period ( 8.0 before vs. 35.5 during, $p=0.022$ ). More consult patients were on anticoagulation ( 8.8 before vs. 29.8 during, $p=0.003$ ) and on renal replacement therapy (RRT) during the pandemic ( 2.6 before vs. 11.4 during, $p=0.003)$. There was a significant decrease in the percentage of monthly pediatric consults from 24.9 to 11.9 ( $p=$ 0.024 ), correlating to a decrease in pediatric patients admitted to the hospital (see "Discussion" section).

\section{Location of consults}

After the onset of the pandemic, there was a significant decrease in the percentages of otolaryngology consults called for patients located in the emergency room (from 30.3 to $13.8 \%, p=0.002$ ) and inpatient floors (from 41.1 to $28.8 \%, p=0.029)$. There was an increase in the percentage of consults called from intensive care units (ICU) or stepdown units (from 31.6 to $54.0 \%, p=0.032$; Fig. 2). These shifts were consistent with the changes and restructuring of hospital inpatient units to accommodate large waves of SARS-CoV-2-positive patients at the time.

\section{Type of consults}

The most common consult category across the entire study period was airway, accounting for $47.6 \%$ of all consults (Fig. 3). Rhinology was the second most common ( $16.5 \%$ of consults), followed by Head and Neck (15.0\% of consults). While these consult categories were the most common both before and after the onset of the pandemic, COVID-19 resulted in a significant shift in the categories and reasons for consults. There was a significant decrease in the monthly number of consults in the categories of Otology (9.4-4.5, $p=0.030)$, Rhinology $(19.3-11.8, p=$ $0.040)$, and Head and Neck $(17.1-11.3, p=0.026)$. There were downward trends in the number of consults in the Oral Cavity/Oropharynx and Facial Trauma categories, but these were not of statistical significance $(p=0.080$ and 0.071 , respectively). In the airway category, there was an

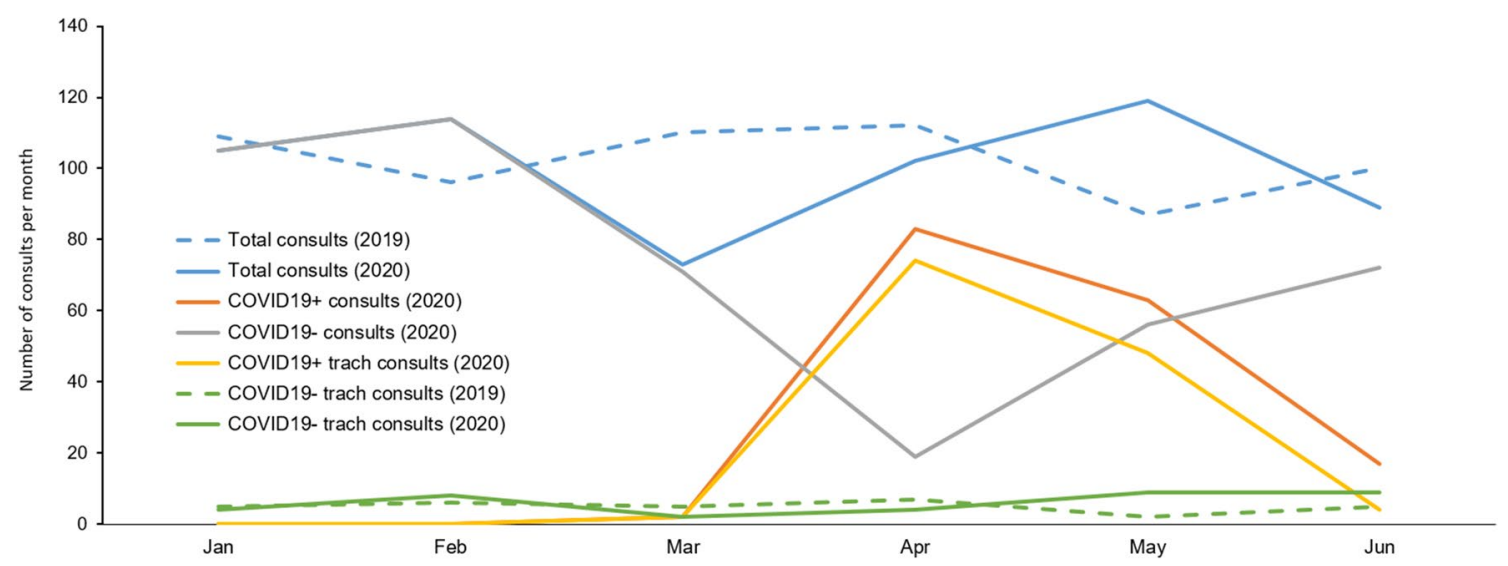

Fig. 1 Timeline of consult volume: absolute number of consults per month in the first 6 months of 2019 and 2020 

of origin of consults before (Jan-Jun 2019, Jan-Feb 2020) COVID-19 pandemic. Asterisk denotes $p<0.05$
Fig. 2 Comparison of location and during (Mar-Jun 2020) the

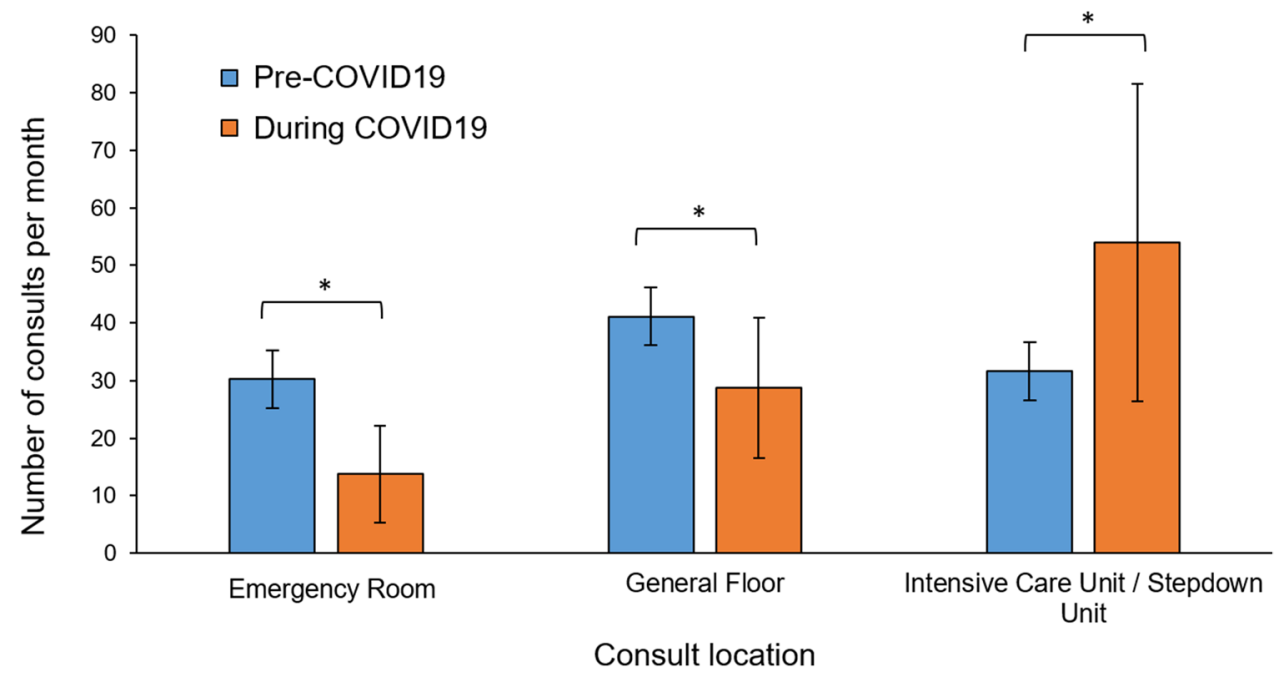

Fig. 3 Comparison of prevalence of consults before (JanJun 2019, Jan-Feb 2020) and during (Mar-Jun 2020) the COVID-19 pandemic, organized by consult categories. Asterisk denotes $p<0.05$

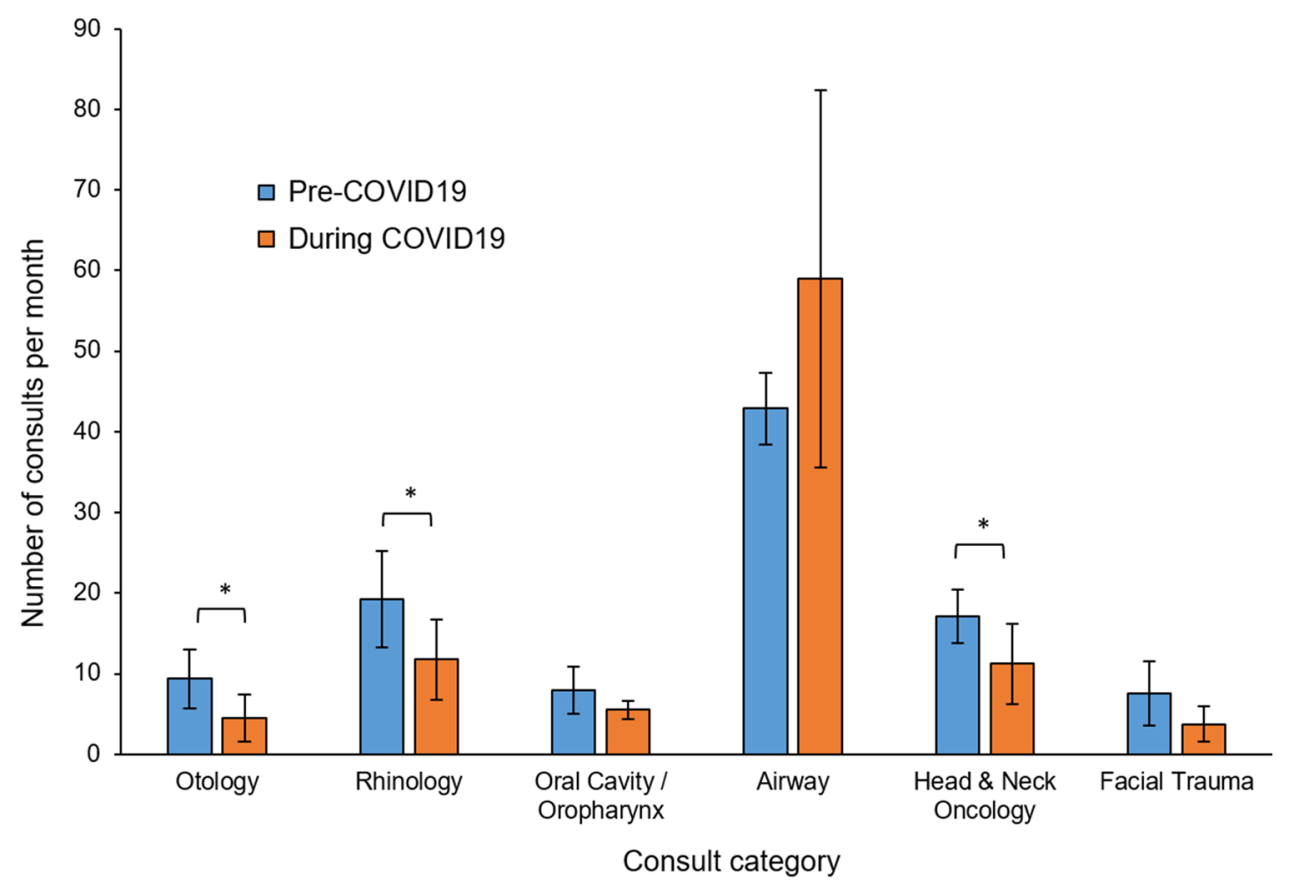

upward trend in the number of monthly consults after the onset of the pandemic, from 42.9 to 59 ( $p=0.058$ ).

Among consults originating in the emergency room, the most common consult category was airway both before (33.1\%) and during (32.1\%) COVID-19 ( $p=0.89)$, and the second most common consult category was Head and Neck both before (23.1\%) and during (28.6\%) COVID-19 $(p=0.39)$. Among consults for hospitalized patients, the most common consult category was airway both before (44.6\%) and during (66.8\%) COVID-19 ( $p<0.0001)$, and the second most common consult category was rhinology both before (19.2\%) and during (12.9\%) COVID-19 ( $p=$ $0.01)$.

\section{Airway and tracheostomy consults}

The onset of the COVID-19 pandemic resulted in a significant increase in the relative incidence of airway consults, from an average of $41.2 \%$ of monthly consults to $61.6 \%$ ( $p=$ 0.02 ). Of the 236 airway consults between March and June of 2020, 152 were TRCs (128 in SARS-CoV-2-positive patients and 24 in SARS-CoV-2-negative patients). Of the TRCs, 105 $(69.1 \%)$ were requests for new tracheostomy and $47(30.9 \%)$ were consults for tracheostomy-related issues, including size adjustments, bleeding, concern for peristomal infection or granulation, and concern for tracheitis. As documented separately, both open and percutaneous tracheostomies were 
Fig. 4 Airway consults. A Comparison of volume of tracheostomy-related consults before (Jan-Jun 2019, Jan-Feb 2020) and during (Mar-Jun 2020) the COVID-19 pandemic. Asterisk denotes $p<0.05$. B Distribution of airway consults in SARS-CoV-2-positive patients during the pandemic
A

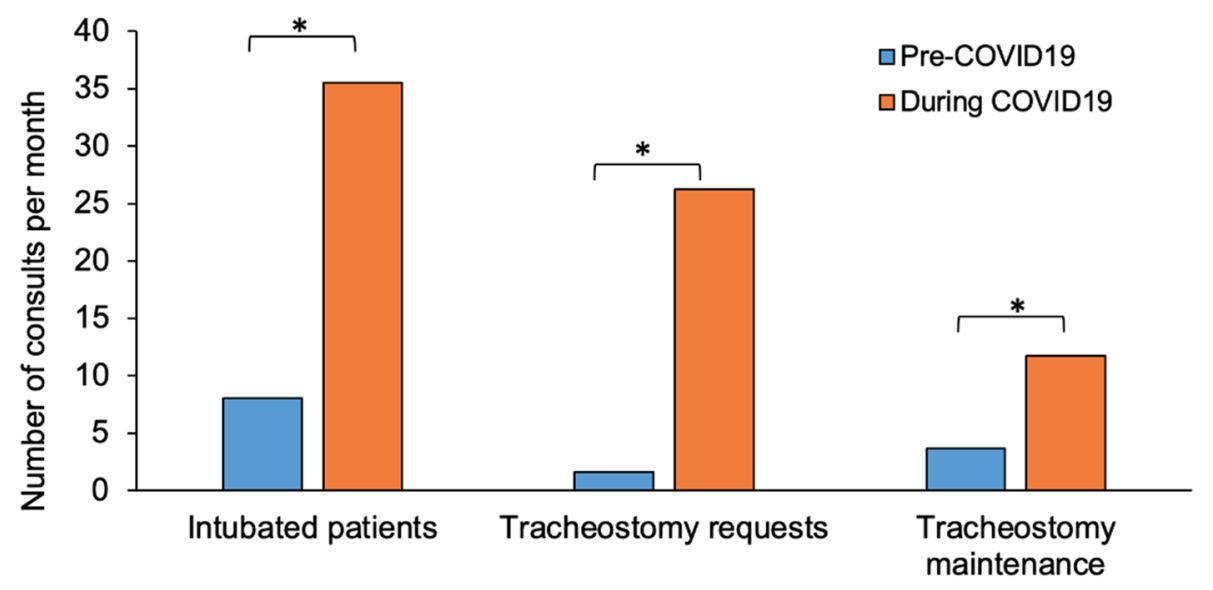

B

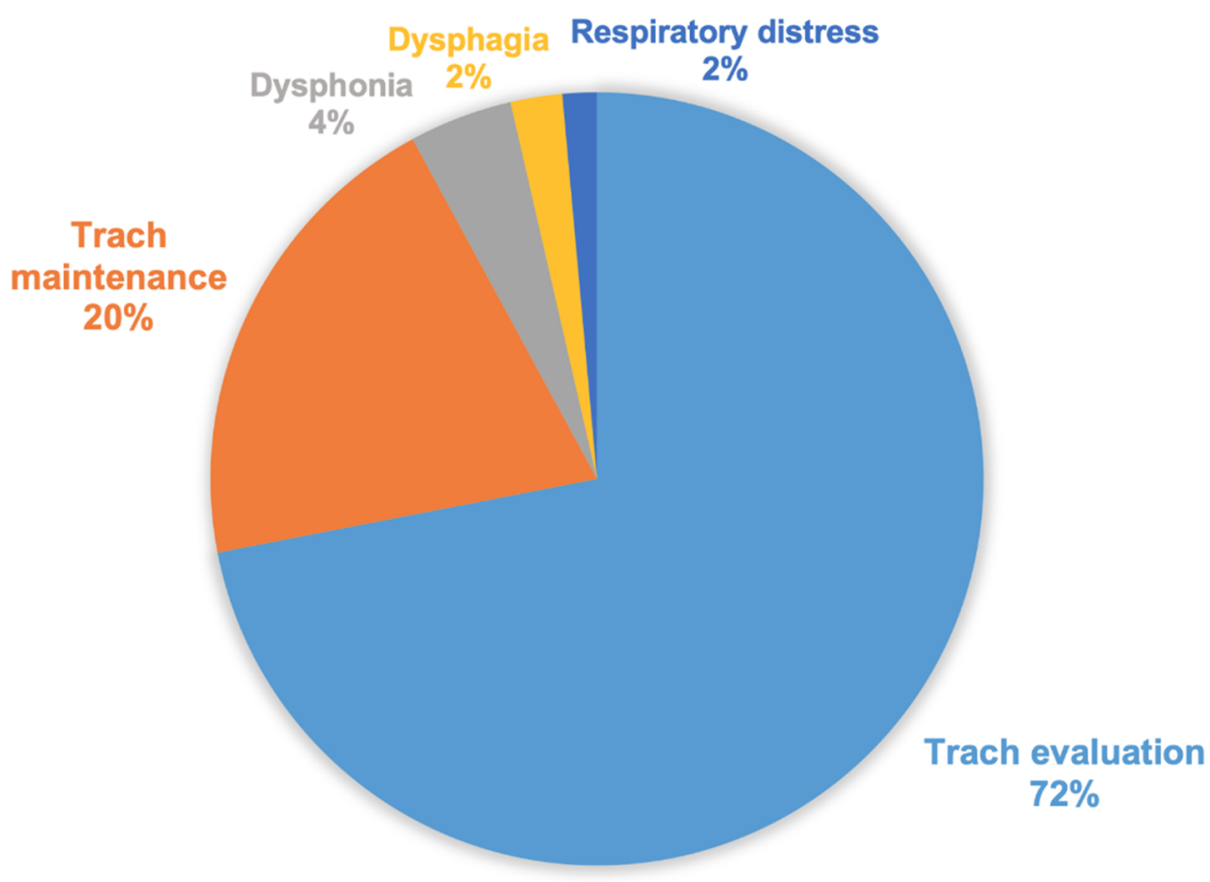

performed at our institution, in traditional ICUs, operating rooms or operating rooms converted to ICUs, after a median intubation time of 24 days [13].

Compared to the pre-COVID-19 period, there was a $4.4 \times$ increase in consults for intubated patients (8.0-35.5 per month; $p=0.02$ ), a $16 \times$ increase in requests for tracheostomy (1.63-26.3 per month, $p=0.02$ ), and a $3.3 \times$ increase in other TRCs (3.6-11.8, $p=0.02$; Fig. 4A).

During the pre-COVID-19 era, the most common reasons for airway consults were respiratory distress (34\%), dysphonia (17\%), dysphagia (17\%), and tracheostomy maintenance (8\%) (Figure S1A). This distribution remained largely unchanged for SARS-CoV-2-negative patients during the COVID-19 period, apart from an increase in the proportion of tracheostomy maintenance consults (from 8 to $21 \%, p=0.0004$; Figure S1B). In SARS-CoV-2-positive patients, there was a dramatic increase in new tracheostomy requests given prolonged intubation in this population (from 4 to $72 \%$ of airway consults, $p<0.0001$; Fig. 4B). Our department's experience with tracheostomies during the COVID-19 pandemic has been previously documented in detail $[4,13]$. 


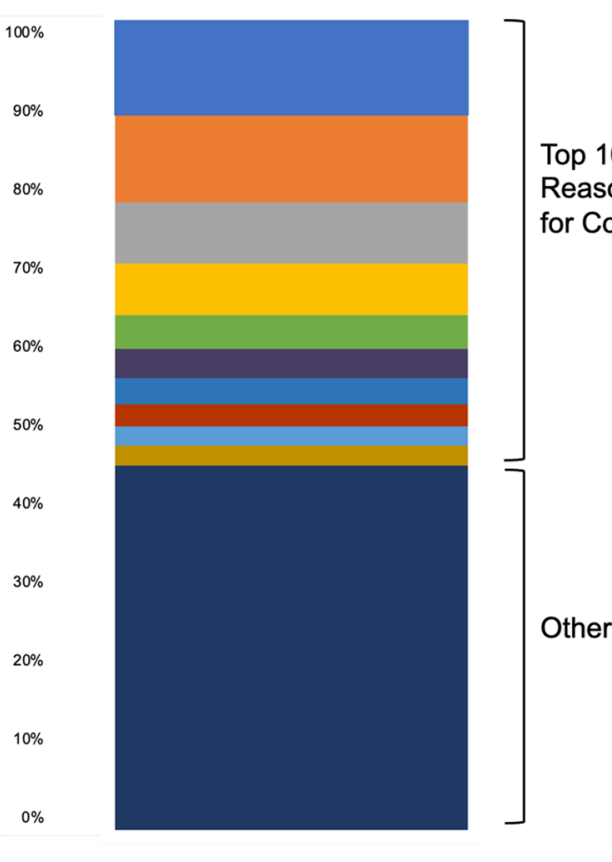

Pre-COVID-19

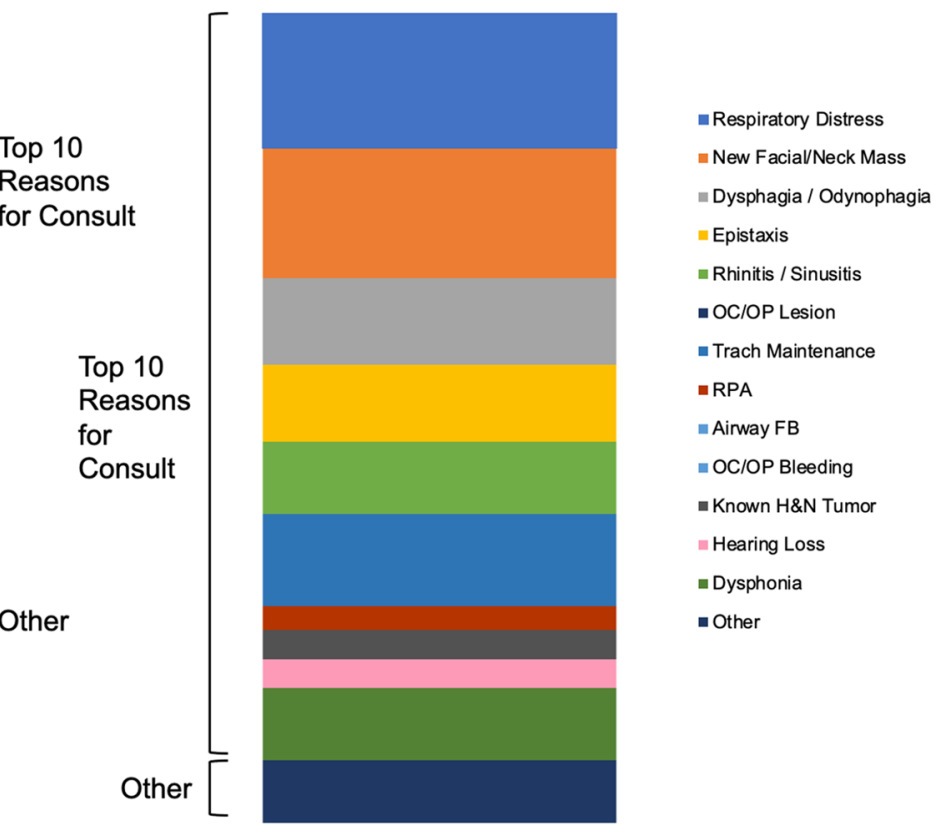

During COVID-19

Fig. 5 Comparison of top ten most common reasons for consult before (Jan-Jun 2019, Jan-Feb 2020) and during (Mar-Jun 2020) the COVID19 pandemic in SARS-CoV-2-negative patients

\section{Consults in SARS-CoV-2-negative patients}

While the majority of consults for SARS-CoV-2-positive patients were in the airway category, consults for SARS$\mathrm{CoV}$-2-negative patients before and during the pandemic spanned multiple categories. The top ten most common reasons for consult in SARS-CoV-2-negative patients before and during the pandemic were similar, with reasons such as respiratory distress, new facial/neck mass, dysphagia/ odynophagia, epistaxis, rhinitis/sinusitis, and trach maintenance ranking near the top in both periods (Fig. 5). In the pre-COVID-19 period, however, the top ten reasons for consult represented $55 \%$ of all consults, whereas during the COVID-19 period, they accounted for $92 \%$ of all consults $(p<0.0001)$. This large difference reflects a significant relative decrease in consults for reasons such as otitis externa, cough, vertigo, nasal bone fractures, ankyloglossia, postoperative concerns, and more, which collectively were heavily represented in the pre-COVID-19 period (Figure S2).

\section{Procedural consults}

Prior to the COVID-19 pandemic, $17.3 \%$ of consults resulted in an operation performed by an otolaryngologist in an operating room or ICU (Table 2). While this number increased to $31.2 \%$ after the onset of the pandemic, this difference was not statistically significant $(p=0.122)$. The percentage of consults requiring endoscopies in the operating room-including flexible or rigid nasal endoscopies, laryngoscopies or bronchoscopies-remained stable after the start of the pandemic, from 2.0 to $1.8 \%$ of all consults ( $p=$ 0.814 ). The percentage of bedside endoscopies-which included flexible fiberoptic laryngoscopies, flexible or rigid nasal endoscopies, and tracheoscopies-decreased significantly after the start of the pandemic, from 55.1 to $29.7 \%$ of consults $(p=0.012)$. The percentage of consults resulting in other bedside procedures remained stable, with control of epistaxis, control of oral cavity or oropharyngeal bleeding, replacement of tracheostomy tube and repair of laceration ranking among the top five most common bedside procedures both before and during the pandemic. Among epistaxis consults in the pre-COVID-19 period, $84.6 \%$ required bedside procedures, including nasal packing or manual pressure, whereas $15.4 \%$ required operative intervention. In the COVID-19 period, $87.5 \%$ required bedside procedures, while $12.5 \%$ required operative intervention $(p=0.49)$.

\section{Discussion}

This is the first study to provide an in-depth characterization of the changes in patterns and trends of otolaryngology inpatient consultations before and during the COVID-19 pandemic in the US. Herein we have reported changes in volume, location, and type of otolaryngology consults, as well as changes in medical, procedural or surgical outcome 
of consults. These changes can be attributed to modifications in the approach towards inpatient consultations by otolaryngology services as well as modifications by consulting inpatient teams requesting otolaryngology evaluation. Additionally, there was a shift in the overall types of pathologies being evaluated by the hospital during the pandemic - an increased number of patients with (COVID-19-related) airway pathologies presented to the hospital, while patients with non-urgent otolaryngologic complaints were more likely to stay at home (per social distancing and quarantine recommendations) or to be referred for outpatient or remote evaluation.

Our analysis of the volume of otolaryngology consults between the periods before and during the COVID-19 pandemic revealed an initial drop in monthly consults in March 2020, followed by a rise in April and May 2020, and ultimately, a return to the normal average number of monthly consults. The nadir seen in March 2020 is likely multifactorial but may be attributed to new local lockdown policies and the hospital's shift in focus with a large ongoing logistical undertaking. Deferment of non-emergent evaluations by both consultants and consulting teams also likely played a role, especially given limited quantities of PPE available. The rapid rise in consults seen in April and May 2020, on the other hand, is explained by the need for tracheotomy in the large population of SARS-CoV-2-positive patients requiring prolonged intubation and ventilation. Most tracheotomies were performed jointly by otolaryngologists and thoracic surgeons at our institution $[4,13]$.

At NYP-Weill Cornell, reconfiguration of physical space and hospital services included the initial separation of patients in the emergency room according to SARS-CoV-2 status and several measures to accommodate the admission of more critically ill adult patients in ICU settings. These measures included the conversion of operating and recovery rooms to ICUs, the creation of new ICUs in adjacent hospital buildings, and the temporary use of pediatric inpatient spaces as ICUs following transfer of most pediatric patients to NYP-Morgan Stanley Children's Hospital of New York. These significant changes in hospital configurations may explain some of the demographic trends in inpatient otolaryngology consultations during the pandemic described in our study: (1) there were very few pediatric consults, (2) significantly more consults originated from ICUs and (3) fewer consults originated from inpatient floors and emergency rooms.

In addition to a shift in the location of consults, a comparison of demographic characteristics of consult patients before and during the COVID-19 pandemic showed differences consistent with the pathophysiology of COVID-19. SARS-CoV-2-positive patients seen between March and June of 2020 had higher rates of anticoagulation for thromboprophylaxis [14] and higher rates of RRT, both of which are expected in the COVID-19 population [15]. SARS-CoV2-positive patients were also significantly more likely to be intubated at the time of consult, which is also expected given the high rates of respiratory failure and ARDS [16]. Not surprisingly, the majority of consults for SARS-CoV2-positive patients were due to prolonged intubation from respiratory failure and ARDS, often requiring tracheotomy. Notably, while there was an increase in otolaryngology airway evaluations during the pandemic, the number of bedside endoscopies drastically decreased, likely due to deferment of airway procedures as per published recommendations during the early stages of the pandemic [8]. Interestingly, while anosmia has been a frequently encountered and widely reported symptom in SARS-CoV-2-positive patients, it was not seen as a primary reason for consult or an isolated symptom in our study. As previously documented, it is possible that anosmia more strongly associates with outpatient clinical courses of COVID-19, potentially representing a milder clinical course that does not warrant hospitalization, and that most presentations of anosmia have been dealt with on an outpatient basis [17].

Unlike consults for SARS-CoV-2-positive patients, otolaryngology consults for SARS-CoV-2-negative patients during the pandemic showed a similar overall pattern to consults before the pandemic. Nonetheless, a comparison of these two groups of consults revealed a few notable differences that may speak to the relative urgency of certain otolaryngologic chief complaints, or at least to how these are perceived by the general population. In both SARSCoV-2-negative patients seen during the pandemic and patients seen before the pandemic, the top ten most common reasons for consult included respiratory distress, dysphagia/odynophagia, epistaxis, and rhinitis/sinusitis. In fact, the top five most common reasons did not change, except for the introduction of trach maintenance during the pandemic period. This suggests that these reasons for consult are part of a core of otolaryngologic chief complaints that non-otolaryngologist physicians may not feel comfortable managing independently, either expectantly or by telemedicine consultation, regardless of pandemic times. However, the fact that the top ten most common reasons for consult represented a much larger proportion of all consults in SARS-CoV-2-negative patients during the pandemic than before the pandemic ( 92 vs. $55 \%$ ) indicates that there was a much wider variety of otolaryngologic chief complaints prior to the pandemic; many of these were likely deemed less urgent or crucial during the pandemic. These included otitis externa, cough, vertigo, and nasal bone fractures. Two exceptions to this rule were hearing loss and evaluation of known head and neck tumors-which are generally seen in outpatient settings - but ranked in the top ten reasons for consult in SARS-CoV-2-negative patients seen during the pandemic. It is possible that these represented patients who 
would have typically been seen in an outpatient setting, but who resorted to the emergency room given the difficulty arranging office appointments during the pandemic.

In addition to the aforementioned potential reasons for changes in otolaryngologic consultation patterns seen at our institution, the COVID-19 pandemic resulted in significant reassignment of staff within our hospital, which may have resulted in redistribution of expertise and skills. For instance, nurses and resident physicians who previously worked on surgical floors were assigned to ICUs, where they were faced with new challenges associated with airway management and may have resorted to otolaryngology consults at higher rates.

Beyond the shifts in consultation patterns revealed in this study, the COVID-19 pandemic had a tremendous impact on the department of otolaryngology at our institution. Over half of otolaryngology residents were redeployed to ICUs for some duration of time, which undeniably resulted in educational tradeoffs such as decreased operative experience and increased exposure to management of critically ill patients. Faculty members experienced a decrease in outpatient inperson visits to $16-35 \%$ of their pre-pandemic baseline, but also a tremendous increase in remote teleconsultations, resulting in a recovery of pre-pandemic outpatient volumes of $>50 \%[18]$.

Our study had several limitations, most notably that our findings are limited to a single institution. The landscape of the COVID-19 pandemic, operations and standardized practices of healthcare centers, and demographics of patient populations drastically vary around the world. Thus, our experience may not necessarily reflect those of institutions in other geographic areas. Additionally, the difference in months of the year included in each studied period could introduce biases into our analysis due to potential seasonal variations.

\section{Conclusion}

As others have described, the COVID-19 pandemic has resulted in significant reductions in the overall volume of procedures and outpatient consultations in otolaryngology $[2,19]$ and in delays in the care of non-COVID-19 otolaryngologic pathologies [20]. Some groups have reported reductions in inpatient and emergency room otolaryngologic consultations in Europe [21, 22]. Our study demonstrates the significant impact of the pandemic on the patterns of otolaryngology inpatient consultations at our institution. Specifically, it suggests that a set of otolaryngologic chief complaints require expedited evaluation and management in an inpatient setting, whereas a larger portion of inpatient consultations may be better suited for outpatient management.
Our experience as a large hospital in an epicenter of the pandemic can help inform other otolaryngology services on how to best prepare and allocate resources to address the COVID-19 pandemic, which may be particularly useful for institutions located in new epicenters of COVID-19. Our results can also help guide our own institution on how to address future pandemic scenarios. Additionally, beyond a pandemic setting, our findings may justify a broader need to create dedicated multidisciplinary airway and tracheotomy teams to face an influx of airway consultations, to adjust otolaryngology resident education to reflect a shift in otolaryngologic pathologies, and to allocate further resources to remote otolaryngologic consultation and optimization of outpatient otolaryngology care.

Supplementary Information The online version contains supplementary material available at https://doi.org/10.1007/s00405-021-06963-7.

Author contributions AS: conceptualization, methodology, formal analysis, investigation, writing — original draft, writing-review and editing, project administration. AC: conceptualization, formal analysis, investigation, writing —original draft. SML: conceptualization, formal analysis, investigation, writing - review and editing. NZF: investigation, writing — review and editing. SSB: investigation, writing — review and editing. MG: investigation, writing-review and editing. KL: investigation, writing - review and editing. DAS: investigation, writing - review and editing. ABT: conceptualization, writing-review and editing, supervision. APS: conceptualization, formal analysis, writing-review and editing, supervision.

Funding None.

Data availability Not applicable.

Code availability Not applicable.

\section{Declarations}

Institutional review board approval Submission Number 20-05022023: patterns of Otolaryngologic Consultation Services Before, During, and After the COVID-19 Pandemic (deemed exempt by Weill Cornell Institutional Review Board).

Conflicts of interest None.

Ethical approval Not applicable.

Consent to participate Not applicable.

Consent for publication Not applicable.

\section{References}

1. Zhou F, Yu T, Du R, Fan G, Liu Y, Liu Z et al (2020) Clinical course and risk factors for mortality of adult inpatients with COVID-19 in Wuhan, China: a retrospective cohort study. Lancet 395:1054-1062. https://doi.org/10.1016/S0140-6736(20)30566-3 
2. Pearlman AN, Tabaee A, Sclafani AP, Sulica L, Selesnick SH, Kutler DI et al (2020) Establishing an office-based framework for resuming otolaryngology care in academic practice during the COVID-19 pandemic. Otolaryngol Head Neck Surg. https://doi. org/10.1177/0194599820955178

3. NYS Health Profiles (2021). https://profiles.health.ny.gov/hospi tal/alpha/letter:N. Accessed 30 May 2021

4. Long SM, Chern A, Feit NZ, Chung S, Ramaswamy AT, Li C et al (2020) Percutaneous and open tracheostomy in patients with COVID-19. Ann Surg. https://doi.org/10.1097/sla.0000000000 004428

5. Kowalski LP, Sanabria A, Ridge JA, Ng WT, de Bree R, Rinaldo A et al (2020) COVID-19 pandemic: effects and evidence-based recommendations for otolaryngology and head and neck surgery practice. Head Neck 42:1259-1267. https://doi.org/10.1002/hed. 26164

6. Kulcsar MA, Montenegro FL, Arap SS, Tavares MR, Kowalski LP (2020) High risk of covid-19 infection for head and neck surgeons. Int Arch Otorhinolaryngol 24:129-130. https://doi.org/10.1055/s0040-1709725

7. Zou L, Ruan F, Huang M, Liang L, Huang H, Hong Z et al (2020) SARS-CoV-2 viral load in upper respiratory specimens of infected patients. N Engl J Med 382:1177-1179. https://doi.org/10.1056/ nejmc 2001737

8. AAO-HNS position statement: otolaryngologists and the COVID19 pandemic. American Academy of Otolaryngology-Head and Neck Surgery. https://www.entnet.org/content/aao-hns-positionstatement-otolaryngologists-and-covid-19-pandemic. Accessed 22 Nov 2020

9. Hussaini AS, Clark CM, Patel AA, Russo ME, Chia SH, Davidson BJ et al (2020) Management of adult inpatient otolaryngologic consultations during the COVID-19 pandemic: a proposed tierbased triage system. Otolaryngol Head Neck Surg 163:330-334. https://doi.org/10.1177/0194599820931011

10. Bann DV, Patel VA, Saadi R, Gniady JP, Goyal N, McGinn JD et al (2020) Impact of coronavirus (COVID-19) on otolaryngologic surgery: brief commentary. Head Neck 42:1227-1234. https://doi.org/10.1002/hed.26162

11. Anagiotos A, Petrikkos G (2020) Otolaryngology in the COVID-19 pandemic era: the impact on our clinical practice. Eur Arch Otorhinolaryngol 1:1. https://doi.org/10.1007/ s00405-020-06161-x

12. Wickemeyer JL, Billings KR, Valika TS (2020) Evolving management of COVID-19: a multi-institutional otolaryngology perspective. Otolaryngol Head Neck Surg 163:259-264. https://doi.org/ $10.1177 / 0194599820930244$
13. Long SM, Feit NZ, Chern A, Cooley V, Hill SS, Rajwani K et al (2021) Percutaneous and open tracheostomy in patients with COVID-19: the Weill Cornell experience in New York city. Laryngoscope. https://doi.org/10.1002/lary.29669

14. Chowdhury JF, Moores LK, Connors JM (2020) Anticoagulation in hospitalized patients with COVID-19. N Engl J Med 383:16751678. https://doi.org/10.1056/nejmclde2028217

15. Yang X, Jin Y, Li R, Zhang Z, Sun R, Chen D (2020) Prevalence and impact of acute renal impairment on COVID-19: a systematic review and meta-analysis. Crit Care 24:356. https://doi.org/10. 1186/s13054-020-03065-4

16. Berlin DA, Gulick RM, Martinez FJ (2020) Severe COVID-19. N Engl J Med. https://doi.org/10.1056/nejmcp2009575

17. Yan CH, Faraji F, Prajapati DP, Ostrander BT, DeConde AS (2020) Self-reported olfactory loss associates with outpatient clinical course in COVID-19. Int Forum Allergy Rhinol 10:821-831. https://doi.org/10.1002/alr.22592

18. Sclafani AP, Shomorony A, Stewart MG, Grunstein E, Overdevest JB (2021) Telemedicine lessons learned during the COVID-19 pandemic: the augmented outpatient otolaryngology teleconsultation. Am J Otolaryngol 42:102960. https://doi.org/10.1016/j. amjoto.2021.102960

19. Kuhar HN, Heilingoetter A, Bergman M, Worobetz N, Chiang T, Matrka L (2020) Otolaryngology in the time of corona: assessing operative impact and risk during the COVID-19 crisis. Otolaryngol Head Neck Surg 163:307-315. https://doi.org/10.1177/01945 99820930214

20. Gallo O, Locatello LG, Orlando P, Martelli F, Bruno C, Cilona $\mathrm{M}$ et al (2020) The clinical consequences of the COVID-19 lockdown: a report from an Italian referral ENT department. Laryngoscope Investig Otolaryngol 5:824-831. https://doi.org/10.1002/ lio2.446

21. Gelardi M, Iannuzzi L, Trecca EMC, Kim B, Quaranta NAA, Cassano M (2020) COVID-19: what happened to all of the otolaryngology emergencies? Eur Arch Otorhinolaryngol 277:3231-3232. https://doi.org/10.1007/s00405-020-06046-z

22. Klimek L, Hagemann J, Alali A, Spielhaupter M, Huppertz T, Hörmann $\mathrm{K}$ et al (2020) The toll of noninfected CRS patients to the COVID-19 pandemic. Rhinol 58:522-523. https://doi.org/10. 4193/Rhin 20.250

Publisher's Note Springer Nature remains neutral with regard to jurisdictional claims in published maps and institutional affiliations. 